Oikos 123: 3-4, 2014

doi: $10.1111 / j .1600-0706.2013 .01242 . x$

(C) 2013 The Authors. Oikos (C) 2013 Nordic Society Oikos

\title{
Dispersal, evolution and range dynamics - a synthesis
}

\author{
Alexander Kubisch and Emanuel A. Fronhofer \\ A. Kubisch (alexander.kubisch@univ-montp2.fr), Inst. des Sciences de l'Evolution (UMR 5554), Univ. Montpellier II, CNRS, Place Eugène \\ Bataillon, FR-34095 Montpellier cedex 5, France. - E. A. Fronhofer, Dept of Aquatic Ecology, Eawag: Swiss Federal Institute of Aquatic Science \\ and Technology, Ueberlandstr. 133, CH-8600 Duebendorf, Switzerland.
}

The distribution of species in space and time is one of the oldest puzzles in ecology. Already Charles Darwin pointed this out over 150 years ago, when he asked: "Who can explain why one species ranges widely and is very numerous, and why another allied species has a narrow range and is rare?" (Darwin 1859). And yet, although much time has passed since 1859 and ecology has experienced an important transition from Natural History to theory based research, we still do not comprehensively understand the formation of geographic ranges for any given species (Gaston 2009). Nevertheless, a lot of effort has been invested into clarifying mechanisms and processes, which affect the formation of species' ranges (Geber 2011). Yet, we feel that important advances in this field have been hampered by the lack of a common framework, especially since range formation is being studied in multiple disciplines and at multiple scales (Gaston 2009, Geber 2011).

In their pioneering survey on the study of futures and particularly the consequences of global changes on the dynamics and function of ecological systems, Coreau et al. (2010) conclude that many of the predictions of future systems that have been made so far may be erroneous due to the complex nature of ecological and evolutionary processes. Many of their interviewees emphasized the problem of dealing with ecological complexity and non-linearity and the resulting multiple future possibilities. When studying the emergence of range borders we are confronted with the very same problem of ecosystem complexity. Nevertheless, with this virtual special issue of Oikos we aim at least at disentangling the complex and interacting ecological and evolutionary forces that are responsible for the formation of species' ranges. In Kubisch et al. (2013) we emphasize the central importance of dispersal for range formation and develop a framework that organizes all eco-evolutionary forces that influence dispersal and therefore also the dynamics of species' ranges hierarchically, including genes, individuals, populations, landscapes and communities. This allows us to dissect the most influential interactions and feedbacks, which create the complexity perceived in natural systems. In the sense of Bonte et al. (2012) we expand this synthesis by compiling a set of publications from previous issues of Oikos, each of which highlights some of the important aspects brought together in our framework.

When investigating species' range formation it is very helpful to take a metapopulation perspective. Note that we here use the term metapopulation in the very broad sense of simply a spatially structured population. For species living in distinct local populations connected by dispersal, the long term survival is determined by colonization and extinction rates. Colonization and extinction rates may change over space due to environmental gradients in habitat characteristics, such as temperature, rainfall or humidity, to name just a few. In such gradients range limits emerge, where the probability of colonization of new habitats is equal to the risk of local population extinction (Oborny et al. 2009). Already Lennon et al. (1997) have shown over 15 years ago that environmental gradients may lead to stable and well defined range boundaries. With their modeling approach, Mustin et al. (2009) present an interesting example of such landscape effects. They show that the velocity of range shifts during climate change, and thus whether a species might track these changes or not, can be strongly influenced simply by the position of the populations inside a habitat gradient.

Clearly, environmental factors like gradients in habitat suitability or connectivity that can be observed at the landscape-level are among the most important determinants of distributional limits. Yet, biotic eco-evolutionary forces can be just as influential. Intraspecific factors like the type of density regulation (e.g. contest or scramble competition) and density-dependent dispersal can affect range shifts and thus even the extinction risk of entire metapopulations (Best et al. 2007). Andersen et al. (2004) investigated densitydependent dispersal in roe deer and found a three to twentyfold increase of the dispersal tendency of individuals in marginal habitats, thus strongly increasing the probability of colonization of new habitats.

Biotic interactions also include interactions between individuals of different species. One of the many important questions in this respect is, whether these interactions, which usually take place at small spatial scales, can alter the dynamics of ranges at large scales. In a theoretical study, 
Singer et al. (2013) compared the influence of mutualistic, commensalistic and parasitic interactions between species on the resulting range dynamics during climate change. They found strong differences between the different types of biotic interactions, leading for example to the conclusion that species suffering from severe parasitism are likely to be most prone to extinction, when environmental conditions change. Aragón and Sánchez-Fernández (2013) used a modeling approach to single-out the effects of predator-prey interactions on species distribution data. These authors could show for the short-toed eagle and its prey that biotic interactions may very well impact the macroscopic distributions of species.

Another level of complexity arises from the interrelation between genotype and phenotype. Monty and Mahy (2010) investigated the evolution of dispersal during a range expansion event of the invasive plant Senecio inaequidens in France. Although they could not detect phenotypic variation in the individual plant's dispersal traits with respect to their distance from the invasion origin, they could show dispersal differences in common garden experiments. Apparently, phenotypic plasticity seems to be very high and is an important mechanism shaping ranges as well.

In addition to dispersal, local adaptation of organisms is also an important determinant of range limits. Alexander and Edwards (2010) reviewed evidence for niche shifts in invasive plants. They list important factors that affect the probability of niche shifts for a given system. Among these are many of the factors, which we also stress to be of outmost importance for range formation, like e.g. dispersal (gene flow) and biotic interactions.

All these factors we mention here bring us back to the question we asked in the beginning: where am I and why? Many factors at all hierarchical levels influence dispersal, colonization and (local) extinction. The resulting complexities and non-linearities are challenging for ecological and evolutionary research. With the present compilation of studies we intend to sensitize scientists working empirically and theoretically not to forget about the interaction of ecology and evolution. As we have noted in the beginning, we still do not comprehensively understand the formation of any given species' range. However, we are convinced that the synthesis of the factors and forces affecting range formation we present in Kubisch et al. (2013) is helpful in advancing our understanding of species' geographic distributions.

\section{References}

Alexander, J. M. and Edwards, P. J. 2010. Limits to the niche and range margins of alien species. - Oikos 119: 1377-1386.

Andersen, R. et al. 2004. When range expansion rate is faster in marginal habitats. - Oikos 107: 210-214.

Aragón, P. and Sánchez-Fernández, D. 2013. Can we disentangle predator-prey interactions from species distributions at a macro-scale? A case study with a raptor species. - Oikos 122: 64-72.

Best, A. S. et al. 2007. Which species will succesfully track climate change? The influence of intraspecific competition and density dependent dispersal on range shifting dynamics. - Oikos 116: 1531-1539.

Bonte, D. et al. 2012. Editorial - synthesis in Ecology. - Oikos 121: 801-803.

Coreau, A. et al. 2010. Exploring the difficulties of studying futures in ecology: what do ecological scientists think? - Oikos 119: 1364-1376.

Darwin, C. 1859. On the origin of species by means of natural selection, or the preservation of favoured races in the struggle for life. - John Murray.

Gaston, K. J. 2009. Geographic range limits: achieving synthesis. - Proc. R. Soc. B 276: 1395-1406.

Geber, M. A. 2011. Ecological and evolutionary limits to species geographic ranges. - Am. Nat. 178: S1-S5.

Kubisch, A. et al. 2013. Where am I and why? Synthesizing range biology and the eco-evolutionary dynamics of dispersal. - Oikos 123: 5-22.

Lennon, J. J. et al. 1997. A metapopulation model of species boundaries. - Oikos 78: 486-502.

Monty, A. and Mahy, G. 2010. Evolution of dispersal traits along an invasion route in the wind-dispersed Senecio inaequidens (Asteraceae). - Oikos 119: 1563-1570.

Mustin, K. et al. 2009. The dynamics of climate-induced range shifting; perspectives from simulation modelling. - Oikos 118: 131-137.

Oborny, B. et al. 2009. Metapopulation dynamics across gradients - the relation between colonization and extinction in shaping the range edge. - Oikos 118: 1453-1460.

Singer, A. et al. 2013. Interspecific interactions affect species and community responses to climate shifts. - Oikos 122: $358-366$. 